\title{
The effectiveness of the Culture for Dientamoeba fragilis from the Stool
}

\author{
Özgür Kurt¹, Nogay Girginkardeşler², Ahmet Özbilgin², Ülgen Zeki Ok² \\ 1 Department of Medical Microbiology, Acibadem University School of Medicine, İstanbul, Turkey \\ 2 Department of Parasitology, Manisa Celal Bayar University School of Medicine, Manisa, Turkey
}

\begin{abstract}
Objective: In the identification of Dientamobea fragilis, Polymerase chain reaction (PCR) is by far more sensitive than microscopic examination of permanent-stained smears, while it is faster and less labour-intensive than culture; however, it is expensive. We aimed to assess the sensitivity of culture and compare the efficacies of three culture media: Robinson's medium (RM), Dobell's medium (DM) and Talis's Medium (TM), for the identification of D. fragilis.

Materials and Methods: Stool samples of 104 individuals admitted to Manisa Celal Bayar University Hospital with complaints such as abdominal pain, itching, meteorism and diarrhea, and no detection of $D$. fragilis in the initial examination of trichrome-stained smears of their stools, were cultivated in RM, DM and TM on the same day.

Results: Trophozoites of D. fragilis were detected in 11 of 104 (10.6\%) samples, where all were positive in RM, while 3 and 4 of 11 were also positive in DM and TM, respectively. Two samples were found to be positive in all three media. RM was by far the leading medium to supply vast numbers of trophozoites.

Conclusion: These results indicate that culture is more effective than microscopic examination of permanent stained smears of stools to identify D. fragilis when PCR is not available, and RM could be the first choice for culture as it supplies huge amounts of trophozoites useful for further molecular studies.
\end{abstract}

Keywords: Dientamoeba fragilis, diagnosis, culture, Robinson's medium, Dobell's medium, Talis's medium.

\section{INTRODUCTION}

D ientamoeba fragilis is an intestinal protozoon classified among the flagellates in habiting in the caecum and colonic lumen of humans (1,2). It has long been regarded as a commensal microorganism until when clinical reports indicate its association with gastrointestinal and dermatological case (3-8). Laboratory diagnosis of D. fragilis infections has long been limited to the microscopic examination of permanent stained fecal smears (Figure 1). Since this method has several disadvantages such as discontinuous shedding feature of $D$. fragilis, its rapid degeneration outside the body and
Corresponding Author: Özgür Kurt

E-mail:

ozgur.kurt@acibadem.edu.tr

Received: March 12, 2019

Accepted: April 10, 2019

Published: May 30, 2019

\section{Suggested Citation:}

Kurt Ö, Girginkardeşler N, Özbilgin A, Ok ÜZ. Choosing Culture for Dientamoeba fragilis Detection in Stool When PCR is not Available: Assessment of its Efficacy and Comparison of Three Culture Media. Infect Dis Clin Microbiol 2019; 1: 34-41.

DOI: $10.5152 /$ idcm.2019.19005 
need for expert microscopist for the evaluation of smears, many laboratories could not offer routine testing for $D$. fragilis. This may be the reason for the lack of concordant prevalence data for $D$. fragilis for decades $(1,4,9)$.

Until the implementation of Polymerase chain reaction (PCR) in parasitology laboratories, culture has long been the most sensitive method to diagnose $D$. fragilis infection, mostly for research projects $(4,10)$. In vitro culture techniques to isolate and identify parasitic organisms have long been regarded as valuable tools, not only for research but also for clinical laboratories (11). Many clinical laboratories do not apply culture for routine diagnosis, because the methods for in vitro culture are often complex, their quality control is difficult, and it may take more than a day for a final decision (12). On the other hand, culture methods are relatively cost-effective and highly sensitive in the identification and isolation of certain parasites, and they are also useful in supplying vast numbers of living organisms for serological, immunological and drug trial studies $(12,13)$.

D. fragilis culture is maintained in the media also used for the cultivation of Entamoeba spp. (10, 13). According to Dobell, D. fragilis is one of the easiest of

\section{HIGHLIGHTS}

- Compared to microscopy, culture is more sensitive and specific to identify many intestinal protozoa, including D. fragilis in fecal samples. In our study, D. fragilis trophozoites were identified in 11 of 104 stool samples which were initially reported as D. fragilis-negative by microscopic examination.

- Comparison of Robinson's medium (RM), Dobell's medium (DM) and Talis's medium (TM) showed that RM is relatively more sensitive and supplies the highest number of trophozoites for further studies.

- Since culture requires at least 48 hours for a definitive result, it is out of scope for diagnostic laboratories; however, it may be selected for research projects in institutions where PCR is not available.

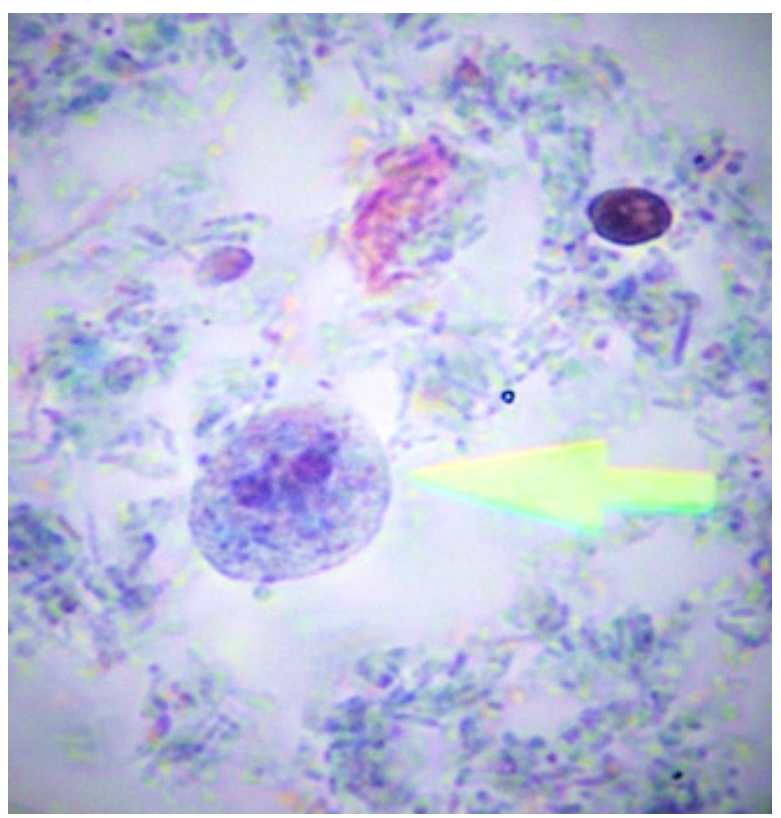

Figure 1. Dientamoeba fragilis trophozoites. (Trichrome stain, x1000; Archive of Manisa Celal Bayar University School of Medicine Department of Medical Parasitology)

the human intestinal amoebae to isolate and grow in vitro (14), thus, it has already been cultivated in several media, such as Dobell's, Boeck-Drbohlav's, Robinson's and Talis's medium (TM), as well as Loeffler's slope medium $(4,6,10,15,16)$. A common feature of these media is being diphasic, including a solid and a liquid phase. The solid phase is mostly made of serum, agar or egg extracts, while the liquid phase contains a combination of solutions. Rice starch is the common carbohydrate source in these media (10). In addition, all are xenic media, where parasite growth is achieved in an unknown composition of microorganisms (12). Among these, RM has been commonly used in the culture of many intestinal parasites including $D$. fragilis (8-10). DM is an old medium including a solid phase of inspissated horse serum slant with an overlay of egg white diluted in Ringer's solution and supplemented with rice starch (10). TM is also a diphasic egg medium developed primarily to obtain Entamoeba histolytica (15). The trials revealed that his formula might supply D. fragilis and Blastocystis spp. in huge numbers as well.

This study aims to compare the efficacies of three different culture media, RM, DM and TM, in the 
Table 1. The formula and preparation of Dobell's Medium.

\begin{tabular}{|l|}
\hline Contents \\
\hline Horse serum \\
\hline Albumin (Egg white) \\
\hline Ringer's solution ( $0.85 \mathrm{gr} \mathrm{NaCl} ; 0.02 \mathrm{gr} \mathrm{CaCl} 2 ; 0.02 \mathrm{gr} \mathrm{CCl}, 100 \mathrm{~mL})$ \\
\hline Preparation \\
\hline Sterilized horse serum was distributed to culture tubes in $3 \mathrm{~mL}$ \\
\hline Tubes were coagulated as inclined at $80^{\circ} \mathrm{C}$ for 2 hours. \\
\hline $\begin{array}{l}\text { An egg white was emulsified in } 500 \mathrm{~mL} \text { of Ringer's solution and } \\
\text { homogenized. }\end{array}$ \\
\hline $\begin{array}{l}\text { The final solution was added onto the inclined coagulated horse } \\
\text { serum to obtain a diphasic culture medium. }\end{array}$ \\
\hline 10 mg of sterilized rice starch was added onto the tube. \\
\hline $\begin{array}{l}\text { All sterile tubes were kept at } 4^{\circ} \mathrm{C} \text { until use, after sterilization control } \\
\text { at } 37^{\circ} \mathrm{C} \text { for one day. }\end{array}$ \\
\hline $\begin{array}{l}\text { A pea-sized or } 5 \mathrm{~mL} \text { of stool sample was inoculated in culture tubes } \\
\text { at room temperature and incubated at } 41^{\circ} \mathrm{C}(14) .\end{array}$ \\
\hline
\end{tabular}

identification of $D$. fragilis in stool samples, in terms of both sensitivity and parasite load.

\section{MATERIALS AND METHODS}

A total of 104 fresh stool samples submitted to Parasitology Laboratory of Manisa Celal Bayar University Hospital and found to be negative for D. fragilis trophozoites after the examination of trichrome-stained smears were enrolled in the study. Their fresh stool samples were inoculated in the culture tubes of RM, DM, and TM. RM and TM were incubated at $37^{\circ} \mathrm{C}$, while DM was incubated at $41^{\circ} \mathrm{C}$, as suggested previously (14). Robinson's medium was prepared and applied as described (10). The contents, preparation, and application of DM and TM were described in Table 1 and Table 2 , respectively. Recovery of $D$. fragilis trophozoites was checked under the light microscope at $\mathrm{x} 400$ magnification, with a drop of culture sediment at 48, 72 and 96 hours after inoculation. Samples with no recovery at $96^{\text {th }}$ hours were considered negative and subcultured once again to avoid false-negative samples, as suggested (17). The subcultures were also examined for reproduction at 48, 72 and 96
Table 2. The formula and preparation of Talis's Medium.

\begin{tabular}{|l|}
\hline Contents \\
\hline $\begin{array}{l}\text { Stock Solution ( } 4.5 \mathrm{gr} \text { Proteose peptone; } 1.8 \mathrm{gr} \mathrm{K} 2 \mathrm{HPO} 4 ; \\
\text { Na2HPO} 4 \text {; } 4.5 \mathrm{gr} \mathrm{grCl} \\
1000 \mathrm{~m} \text { distilled water) }\end{array}$ \\
\hline Dextrose solution $50 \%$ \\
\hline One egg \\
\hline Bovine albumin $5 \%$ \\
\hline Acriflavine $0.6 \%$ \\
\hline Preparation \\
\hline pH of the stock solution was set to 7.6 before sterilization. \\
\hline $\begin{array}{l}\text { The solid phase was prepared by mixing the egg content with } 25 \\
\mathrm{~mL} \text { of stock solution and } 0.08 \mathrm{~mL} \text { of } 50 \% \text { dextrose solution. }\end{array}$ \\
\hline $\begin{array}{l}\text { The mixture was homogenized, distributed to culture tubes in } 1 \mathrm{~mL} \\
\text { and coagulated as inclined at } 80^{\circ} \mathrm{C} \text { for } 2 \text { hours. }\end{array}$ \\
\hline $\begin{array}{l}\text { The liquid phase was prepared by mixing } 100 \mathrm{~mL} \text { of stock solution } \\
\text { with } 2 \mathrm{~mL} \text { of } 5 \% \text { of bovine albumin and } 2 \mathrm{~mL} \text { of } 0.6 \% \text { of acriflavine, } \\
\text { and sterilized. }\end{array}$ \\
\hline $\begin{array}{l}\text { The medium was ready after adding } 2 \mathrm{~mL} \text { of liquid phase onto the } \\
\text { solid phase in the tubes. } 10 \mathrm{mg} \text { of sterilized rice starch was added } \\
\text { into the tubes before inoculation of the stool sample. }\end{array}$ \\
\hline
\end{tabular}

hours after inoculation. Trichrome-stained smears of the cultures were then examined for confirmation of the positives.

The number of $D$. fragilis trophozoites, in each examination field, were classified under x400 magnification as follows: (+) one to three trophozoites; $(++)$ three to ten trophozoites; (+++) more than ten trophozoites. The sensitivity and specificity values of Dobell's medium and TDEM were assessed compared to Robinson's medium.

Manisa Celal Bayar University, School of Medicine Ethics Committee approved the study (Registration number: 2003-49). Written informed consent of the participants was obtained following a detailed explanation of the study protocol.

\section{Statistical analysis}

Chi-squared tests (with Yates' correction) and Fisher's exact test were employed for comparison of categorical variables. The Sensitivity and specificity of TM and DM were calculated with comparison to RM. The data analyses were done using Statistical Package for Social Sciences version $10.0^{\circledR}$ (SPSS 
Table 3. The assessment of the efficacies of Dobell's medium and Talis's medium, compared to Robinson's medium.

\begin{tabular}{|c|c|c|c|c|c|}
\hline & & \multicolumn{3}{|c|}{$\begin{array}{l}\text { Robinson's } \\
\text { medium }\end{array}$} & \\
\hline & & $(+)$ & $(-)$ & Total & \\
\hline \multirow{3}{*}{$\begin{array}{c}\text { Dobell's } \\
\text { medium } \\
\text { (DM) }\end{array}$} & $(+)$ & 3 & 0 & 3 & \multirow{3}{*}{$\begin{array}{c}\text { Sensitivity: } \\
27.3 \% \\
\text { Specificity: } \\
100 \%\end{array}$} \\
\hline & $(-)$ & 8 & 93 & 101 & \\
\hline & Total & 11 & 93 & 104 & \\
\hline \multirow{3}{*}{$\begin{array}{l}\text { Talis's } \\
\text { Medium } \\
\text { (TM) }\end{array}$} & $(+)$ & 4 & 0 & 4 & \multirow{3}{*}{$\begin{array}{c}\text { Sensitivity: } \\
36.4 \% \\
\text { Specificity: } \\
100 \%\end{array}$} \\
\hline & $(-)$ & 7 & 93 & 100 & \\
\hline & Total & 11 & 93 & 104 & \\
\hline
\end{tabular}

Inc.; Chicago, IL, USA), and statistical significance was set as $<0.05$.

\section{RESULTS}

The mean age of the participants age of the participants was $22.2( \pm 6.0$; age range $3-79)$, and there were more females $(n=55 ; 52.9 \%)$ in the study group. Three of every four participants $(\mathrm{n}=78 ; 75.0 \%)$ were referred to parasitology laboratory from Gastroenterology department of internal medicine and paediatrics, followed by dermatology ( $\mathrm{n}=16 ; 15.4 \%)$ and allergy $(10,9.6 \%)$ departments. One-third of the participants $(\mathrm{n}=34 ; 32.7 \%)$ described no symptoms, while abdominal pain and diarrhea were the most common symptoms followed by itching and/or urticaria (19 and 17 individuals, respectively) (Table 3).

Assessment of the study group showed trophozoites of D. fragilis were detected in 11 of 104 (10.6\%) stool samples. All 11 samples were successfully cultivated in RM, while 3 and 4 of these 11 samples were positive in DM and TM, respectively (Table 4). Two samples were cultivated in all media. Trophozoites of $D$. fragilis were identified as round, rice-starch containing organisms, developing leaf-like pseudopodia after 10 minutes at room temperature (Figure 2). All positive samples were confirmed with microscopic examination of their trichrome-stained smears. More parasites were identified in RM tubes, even after 96 hours (Table 5). The sensitivity and values of both $\mathrm{DM}$ and TM compared to RM were shown in Table 4.

More than half of the stool samples had no parasites detected during the routine examination ( $\mathrm{n}=$ 58; 55.8\%). The leading parasite was Blastocystis spp. $(\mathrm{n}=21 ; 20.2 \%)$, followed by Enterobius vermicularis $(\mathrm{n}=6 ; 5.8 \%)$. Among the culture-positive samples, 6 of 11 RM, 2 of 4 TM and all 3 DM-positive samples had Blastocystis spp. (Table 5).

\section{DISCUSSION}

According to some authors, the detection rate of D. fragilis has been improved dramatically by culture $(18,19)$. In a comparative study of diagnostic methods of protozoal diseases with 1.066 patients, the incidence of $D$. fragilis was found to be almost $2 \%$ and $39.3 \%$ by microscopy alone and microscopy with culture, respectively (17). Stool culture was found to be less labour-intensive than trichrome staining plus the isolate can also be used for molecular typing and cryopreservation; it was, therefore, suggested that it should be used to diagnose D. fragilis infection $(16,18,19)$. Using microscopy alone, the detection rate of $D$. fragilis was found to be $3.3 \%$ in the Parasitology Laboratory of Manisa Celal Bayar University in 2003; this figure rose to

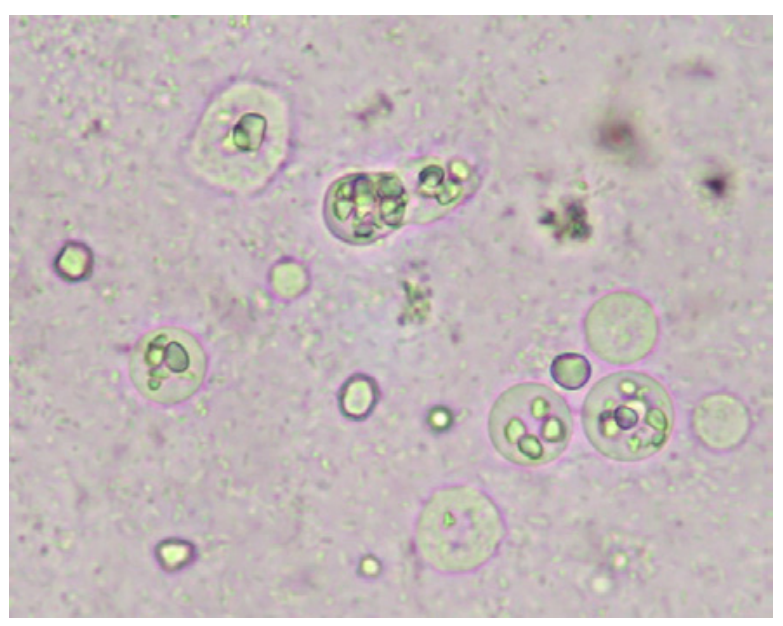

Figure 2. Dientamoeba fragilis trophozoites in culture. Note that D. fragilis trophozoites ingested rice starch while Blastocystis spp. not. Confirmation with microscopic examination of trichromestained smears are required (x400; Archive of Manisa Celal Bayar University School of Medicine Department of Medical Parasitology) 
Table 4. Characteristics of the patients, who were culture-positive.

\begin{tabular}{|c|c|c|c|c|c|c|c|}
\hline NO & Age & Gender & Complaints & Other Parasites & RM & RM & $\mathrm{TM}$ \\
\hline 1 & 25 & $\mathrm{~F}$ & Itching and urticaria & Blastocystis & + & + & + \\
\hline 2 & 8 & M & $\begin{array}{l}\text { Abdominal pain and } \\
\text { constipation }\end{array}$ & none & + & $\mathrm{N}$ & $\mathrm{N}$ \\
\hline 3 & 10 & M & Weight loss & Blastocystis & + & $\mathrm{N}$ & $\mathrm{N}$ \\
\hline 4 & 9 & $\mathrm{~F}$ & Itching and urticaria & none & + & N & $\mathrm{N}$ \\
\hline 5 & 12 & $\mathrm{~F}$ & $\begin{array}{l}\text { Abdominal pain and } \\
\text { constipation }\end{array}$ & Blastocystis & + & + & + \\
\hline 6 & 6 & M & Abdominal pain and diarrhea & $\begin{array}{l}\text { Enterobius } \\
\text { vermicularis }\end{array}$ & + & N & $\mathrm{N}$ \\
\hline 7 & 11 & $\mathrm{~F}$ & $\begin{array}{l}\text { Abdominal pain and } \\
\text { constipation }\end{array}$ & Blastocystis & + & N & $\mathrm{N}$ \\
\hline 8 & 4 & $\mathrm{~F}$ & Abdominal pain and diarrhea & none & + & $\mathrm{N}$ & + \\
\hline 9 & 8 & $\mathrm{~F}$ & $\begin{array}{l}\text { Abdominal pain and } \\
\text { constipation }\end{array}$ & Blastocystis & + & N & $\mathrm{N}$ \\
\hline 10 & 7 & M & Abdominal pain and diarrhea & Giardia lamblia & + & $\mathrm{N}$ & + \\
\hline 11 & 24 & M & Flatulence & Blastocystis & + & + & $\mathrm{N}$ \\
\hline
\end{tabular}

Table 5. Comparison of three culture media in terms of parasite load in the culture tubes.

\begin{tabular}{|c|c|c|c|c|c|c|c|c|c|c|}
\hline \multirow{3}{*}{ Culture Medium } & \multicolumn{4}{|c|}{ Robinson's } & \multicolumn{3}{|c|}{ Dobell's } & \multicolumn{3}{|c|}{ Talis's } \\
\hline & $\begin{array}{l}\text { 气ू } \\
\text { 옴 }\end{array}$ & $48^{\text {th }}$ & $72^{\text {th }}$ & $96^{\text {th }}$ & $48^{\text {th }}$ & $72^{\text {th }}$ & $96^{\text {th }}$ & $48^{\text {th }}$ & $72^{\text {th }}$ & $96^{\text {th }}$ \\
\hline & & $n$ & $\mathrm{n}$ & $\mathrm{n}$ & $\mathrm{n}$ & $\mathrm{n}$ & $\mathrm{n}$ & $\mathrm{n}$ & $\mathrm{n}$ & $\mathrm{n}$ \\
\hline No trophozoites & & 0 & 0 & 0 & 8 & 8 & 8 & 7 & 7 & 7 \\
\hline $1-3^{\star}$ trophozoites & & 0 & 0 & 0 & 1 & 1 & 0 & 1 & 1 & 0 \\
\hline 3-10* trophozoites & & 4 & 4 & 3 & 2 & 2 & 2 & 2 & 2 & 1 \\
\hline$>10^{*}$ trophozoitess & & 7 & 6 & 6 & 0 & 0 & 0 & 1 & 1 & 1 \\
\hline TOTAL n (\%) & \multicolumn{4}{|c|}{$11(10.6)$} & \multicolumn{3}{|c|}{$3(2.9)$} & \multicolumn{3}{|c|}{$4(3.8)$} \\
\hline
\end{tabular}


$5.2 \%$ after the addition of culture even just to individual patients (unpublished data).

Despite regarded as a sensitive and specific method, culture was not common in routine diagnosis of intestinal parasites due to its being labour-intensive and time-consuming $(4,10,17)$. After PCR protocols were launched to detect $D$. fragilis, it has been noticed that old methods have dramatically underestimated its prevalence; for example, in a Danish study involving PCR examination of 22,000 fecal samples of individuals in a period of three years, $D$. fragilis prevalence was found to be $43 \%$ in general population, while it was reaching $71 \%$ in children (20). However, PCR is still an expensive method for many laboratories as it requires a high-standard molecular laboratory, expensive equipment and regular supply of consumable products. The costs of conventional PCR and Real-Time PCR were calculated as 15 USD and 26.5 USD, while the cost of microscopy was 3.5 USD per stool sample (6). In our experience, the long-term costs of culture methods were almost the same compared to microscopic examinations of permanent stained smears and significantly cheaper than PCR. Therefore, culture is a reasonable option for laboratories with limited financial resources.

Successful cultivation of D. fragilis has already been managed in several xenic media, including RM, DM, TM, Boeck-Drbohlav's medium and Loeffler's slope medium $(4,10,15,16)$. Cultivation in axenic medium, where the microbiological content of the medium is predetermined, is essential for all kinds of studies, especially for the ones targeting the virulence and antimicrobial efficacy testing; however, cultivation of $D$. fragilis in an axenic culture medium has not been maintained yet, and this is a significant drawback for antimicrobial efficacy trials and studies on its pathogenicity $(4,21)$.

The xenic medium developed by Robinson in 1968 has been the most preferred medium for the cultivation of E. histolytica, as well as the other species of Entamoeba, Endolimax nana, Blastocystis spp. and D. fragilis $(10 ; 13)$. It is labour-intensive and requires various chemicals that are used to prepare fresh solutions. In some D. fragilis-positive cultures, excessive reproduction of Blastocystis spp. were also noticed in RM, which were harmful to the maintenance of $D$. fragilis trophozoites (10). It may not be a problem in our study group as more than half D. fragilis-positive samples in RM were Blastocystis-positive as well. Although successful in shortterm culture studies, Robinson's medium seems unsuccessful for long-term culture of D. fragilis (19).

Dobell's medium has been used successfully for the culture of Entamoeba spp. and D. fragilis (14). An important detail about successful cultivation in $\mathrm{DM}$ is that the incubation should be at $41^{\circ} \mathrm{C}$, not at $37^{\circ} \mathrm{C}$ (14). In our experience, preparation of Dobell's medium was a long and complicated process, and our results yielded its lower sensitivity in the recovery of $D$. fragilis trophozoites. On the other hand, no excessive reproduction of Blastocystis spp. isolates was noted in DM tubes.

This is probably the first study that used TM to identify D. fragilis in stool samples. It was developed to culture Entamoeba histolytica, but D. fragilis was also cultivated successfully in the trials (15). Preparation of TM was relatively easier compared to RM and DM, but its sensitivity was found to be poorer. Despite the presence of acriflavine in its formula, excessive reproduction of Blastocystis spp. was noted in TM tubes, as in RM.

Table 6. Other parasites identified during the routine examination.

\begin{tabular}{|l|c|c|}
\hline Name of the Parasite & $\mathrm{n}$ & $\%$ \\
\hline No parasite & 58 & 55.77 \\
\hline Blastocystis spp. & 21 & 20.19 \\
\hline Enterobius vermicularis & 6 & 5.76 \\
\hline Entamoeba histolytica/dispar & 4 & 3.85 \\
\hline Endolimax nana & 4 & 3.85 \\
\hline Iodomoeba butschlii & 4 & 3.85 \\
\hline Giardia lamblia & 4 & 3.85 \\
\hline Taenia saginata & 1 & 0.96 \\
\hline Entamoeba coli & 2 & 1.92 \\
\hline
\end{tabular}


The detection of protozoa may be obscured due to limited growth in initial culture tubes; therefore at least two passages should be performed in clinically suspected cases of dientamoebiasis. It was reported that the detection rate of culture among patients with known D. fragilis infection was $40 \%$ and $80 \%$ after the first and second passages, respectively (17). In our study, 2 of the 11 positive samples in Robinson's medium and none in the other media were diagnosed in the second passages.

Our findings indicate that culture is a useful alternative to PCR for the identification of $D$. fragilis in stool samples. It takes at least two days to decide whether there is D. fragilis in the stool, which makes it an unfavorable diagnostic method for routine laboratories, while it provides vast numbers of tro- phozoites, which is essential for research projects involving serological and molecular assessments for D. fragilis. Of the three culture media, Robinson's medium was the most sensitive and reliable one, although it was more labour-intensive compared to DM and TM. The highest number of trophozoites were recovered even at the $96^{\text {th }}$ hour after inoculation in RM, compared to DM and TM.

In conclusion, culture method is still a cost-effective method that may be preferred in laboratories with limited financial resources where PCR is not available. Considering the obscurities about its pathogenicity, anti-microbial response and genotypic as well as the proteomic profile of $D$. fragilis, culture is a good option to obtain piles of trophozoites for further assessments.
Ethics Committee Approval: Manisa Celal Bayar University, School of Medicine Ethics Committee approved the study (No: 2003-49).

Informed Consent: Written informed consent was obtained from the patients who participated in this study.

Peer-review: Externally peer-reviewed.

Author Contributions: Concept - Ö.K., N.G., A.Ö., Ü.Z.O.; Design - Ö.K., N.G., A.Ö., Ü.Z.O.; Supervision - Ö.K., N.G., A.Ö., Ü.Z.O.; Resources - Ö.K., N.G., A.Ö., Ü.Z.O.; Materials - Ö.K., N.G., A.Ö., Ü.Z.O.; Data Collection and/or Processing - Ö.K., N.G., A.Ö., Ü.Z.O.; Analysis and/or Interpretation - Ö.K., N.G., A.Ö., Ü.Z.O.; Literature Search - Ö.K., N.G., A.Ö., Ü.Z.O.; Writing Manuscript - Ö.K., N.G., A.Ö., Ü.Z.O.; Critical Reviews - Ö.K., N.G., A.Ö., Ü.Z.O.

Acknowledgements: We would likte to thank to Mr. İker Pınar, the laboratory technician of the Parasitology LAboratory in Manisa Celal Bayar University Hospital for his cordial contribution to this study. We are also grateful to Dr. C. Graham Clark from London School of Hygiene and Tropical Medicine in London, and Dr. Jeffrey Windsor from Aberystwyth Public Health Laboratory in Wales, UK, fror their sincere support throughout the study, and detailed revision of this manuscript.

Conflict of Interest: The authors have no conflict of interest to declare.

Financial Disclosure: This study derived from the project "The role of culture method in the diagnosis of dientamoebiasis and efficacies of some antimicrobials in its treatment" funded by Manisa Celal Bayar University Department of Scientific Research Projects (2001-031)

\section{REFERENCES}

1 Girginkardesler N, Coskun S, Ok ÜZ. Dientamoeba fragilis: A neglected cause of diarrhea (Article in Turkish). Acta Parasitol Turc 2000; 24: 426-30.

2 Stark D, Barratt J, Chan D, Ellis JT. Dientamoeba fragilis, the Neglected Trichomonad of the Human Bowel. Clin Microbiol Rev 2016; 29: 553-80.

3 Cuffari C, Oligny L, Seidman EG. Dientamoeba fragilis masquerading as allergic colitis. J Pediatr Gastroenterol Nutr 1998; 26: 16-20.

4 Johnson EH, Windsor JJ, Clark CG. Emerging from obscurity:
Biological, clinical and diagnostic aspects of Dientamoeba fragilis. Clin Microbiol Rev 2004; 17: 553-70.

5 Kurt O, Girginkardesler N, Balcioglu IC, Özbilgin A, Ok ÜZ. A comparison of metronidazole and single-dose ornidazole for the treatment of dientamoebiasis. Clin Microbiol Infect 2008; 14: 601-4.

6 Stark D, Barratt J, Roberts T, Marriott D, Harkness J, Ellis J. A review of the clinical presentation of dientamoebiasis. Am J Trop Med Hyg 2010; 82: 614-9. 
7 Miguel L, Salvador F, Sulleiro E, Sánchez-Montalvá A, Molina-Morant D, López I, et al. Clinical and Epidemiological Characteristics of Patients with Dientamoeba fragilis Infection. Am J Trop Med Hyg 2018; 99: 1170-3.

8 Sivcan E, Charyyeva A, Ceylan ŞS, Yürük M, Erdoğan E, Şahin İ. Dientamoeba fragilis infection in patients with gastrointes tinal system complaints [Article in Turkish]. Mikrobiyol Bul 2018; 52: 166-79.

9 Calderaro A, Buttrini M, Montecchini S, Rossi S, Piccolo G, Arcangeletti MC, et al. MALDI-TOF MS as a new tool for the identification of Dientamoeba fragilis. Parasit Vectors 2018; 11: 11.

10 Clark CG, Diamond LS. Methods for cultivation of luminal par asitic protists of clinical importance. Clin Microbiol Rev 2002; 15: $329-41$

11 Ash LR, Orihel TC, editors. Parasite cultures and animal inoculations. Parasites: A guide to laboratory procedures and identification. Chicago: ASCP Press; 1987.p.117-24.

12 Garcia LS, Bruckner DA. Parasite recovery: Culture methods, animal inoculation and xenodiagnosis. In: Diagnostic medical parasitology. Washington: ASM publications, 1993.p.595-603.

13 Daldal N, Taylan Özkan A. Parasite Cultures (In Turkish). Korkmaz M, Ok ÜZ, editors. Laboratory in Parasitology. Izmir: Turkish Parasitology Association Publications, No: 23. 2011.p.87117.

14 Dobell C. Researches on the intestinal protozoa of monkeys and man. X. The life of history of Dientamoeba fragilis: ob- servations, experiments and speculations. Parasitology 1940; 32: 417-61.

15 Talis B, Stein B, Lengy J. Dientamoeba fragilis in human feces and bile. Israel J Med Sci 1971; 7: 1063-9.

16 Barratt JL, Banik GR, Harkness J, Marriott D, Ellis JT, Stark D. Newly defined conditions for the in vitro cultivation and cryopreservation of Dientamoeba fragilis: new techniques set to fast track molecular studies on this organism. Parasitology 2010: 1867-78.

17 Ockert G. Symptomatology, pathology, epidemiology and diagnosis of Dientamoeba fragilis. Trichomonads parasitic in humans. Honinberg BM, editor. New York: Springer, 1990: 394-410.

18 Sawangjaroen N, Luke R, Prociv P. Diagnosis of faecal culture of Dientamoeba fragilis infections in Australian patients with diarrhoea. Trans R Soc Trop Med Hyg 1993; 87: 163-5.

19 Windsor JJ, Macfarlane L, Hughes-Thapa G, Jones SKA, Whiteside TM. Detection of Dientamoeba fragilis by culture. Brit J Biomed Sci 2003; 60: 79-83.

20 Röser D, Simonsen J, Nielsen HV, Stensvold CR, Mølbak K. Dientamoeba fragilis in Denmark: epidemiological experience derived from four years of routine real-time PCR. Eur J Clin Microbiol Infect Dis 2013; 32: 1303-10.

21 Chan THF, Guan MXU, Mackenzie MR. Application of indirect immunoflorescence to detection of Dientamoeba fragilis trophozoites in fecal specimens. J Clin Microbiol 1993; 31: 1710-4. 